Gastroenterologe 2015 $10: 461-463$ DOI 10.1007/s11377-015-0025-9

Online publiziert: 16 . Oktober 2015

๑) Springer-Verlag Berlin Heidelberg 2015

CrossMark

\author{
M. Schumann ${ }^{1} \cdot$ W. Fischbach ${ }^{2} \cdot$ B. Siegmund \\ ${ }^{1}$ Medizinische Klinik I m.S. Gastroenterologie, Infektiologie und Rheumatologie, Charité - \\ Universitätsmedizin Berlin, Campus Benjamin Franklin, Berlin, Deutschland \\ ${ }^{2}$ Medizinische Klinik II, Klinikum Aschaffenburg-Alzenau, Aschaffenburg, Deutschland
}

\title{
Zöliakie und Darmlymphome
}

Die Zöliakie ist nicht nur für Forscher des mukosalen Immunsystems eine wichtige Erkrankung. $\mathrm{Zu}$ diesem Schluss muss man auch hierzulande spätestens seit der Veröffentlichung der Prävalenzdaten der Zöliakie in Deutschland kommen, die kürzlich in der Zeitschrift Deutsches Ärzteblatt erschienen sind [1]. Diese Daten zeigen, dass die Zöliakie mit einer Prävalenz von $0,9 \% \mathrm{zu}$ den häufigsten chronischen Dünndarmerkrankungen gehört und dass diese Prävalenz - entgegen kleinerer Vorstudien - durchaus mit der Prävalenz europäischer Nachbarländer vergleichbar ist. Dabei bleibt jedoch - trotz der aktuellen Publikation - das zentrale Dilemma: Nur etwa jeder Zehnte von Zöliakie Betroffene wird tatsächlich diagnostiziert. Die Patienten, bei denen schlussendlich eine Zöliakiediagnose gestellt wurde, berichten oftmals von einer mehrjährigen Odyssee, die der Erstdiagnose vorausging.

Was sind die Ursachen für diese erhebliche Diskrepanz? Zum einen ist für viele praktizierende Ärzte die Zöliakie weiterhin eine Erkrankung des Kindesalters. Bei Erwachsenen - auch bei solchen, die die sog. 2. Lebenshälfte erreicht haben - wird trotz Vorhandensein eines typischen klinischen Erscheinungsbilds seltener eine Zöliakie differenzialdiagnostisch erwogen. Epidemiologische Daten weisen aber auf einen hohen Anteil an Erwachsenen unter den Erstdiagnostizierten hin [2]. Des Weiteren ist die Diagnosestellung einer Zöliakie oftmals schwierig, da sich in der Mehrzahl der Zöliakiefälle diese nur oligosymptomatisch oder gar komplett subklinisch darstellt. Das bedeutet, dass das sekundär zur Zöliakie bestehende Malabsorptionssyndrom sehr unterschiedliche Ausprägungsformen kennt und zumeist statt einer „typischen Symptomatik“ mit chronischer Diarrhoe, Gewichtsverlust, einer Anämie- und einem Osteoporose-assoziierten klinischen Befund allenfalls einzelne Symptome bestehen. Ein 3. Problem hat sich erst kürzlich in den USA entwickelt und kommt zusehends aus Nordamerika nach Europa: Laut einer Umfrage sind etwa $25 \%$ der US-Bevölkerung der Ansicht, glutenfreie Kost sei gesünder als eine konventionelle Kost und $11 \%$ versuchen ihren Idolen, wie Gwyneth Paltrow oder Lady Gaga, zu folgen und steigen auf eine glutenfreie oder zumindest glutenverminderte Diät um [3]. Dieser ernährungsphysiologisch nicht gut begründbare „Hype“ ist inzwischen zu einem lästigen Alltagsproblem in Zöliakieambulanzen angewachsen, da die routinemäßig verwendeten Zöliakiediagnostika bei Patienten, die bereits vor Monaten beschlossen hatten, glutenfrei zu leben, nicht greifen.

Es gibt jedoch auch einige neue „Chancen“ im Management der Zöliakie - auch diagnostische. So wurde der genetische Hintergrund der Zöliakie eingehend untersucht. Das erhebliche Risiko erstgradig Verwandter eines von Zöliakie Betroffenen, auch an Zöliakie zu erkranken (15\%), sollte genutzt werden: Jeder erstgradig Verwandte sollte einer Zöliakiediagnostik zugeführt werden. Ebenso nutzen bereits viele Endokrinologen die genetisch nachweisbare Artverwandtschaft der Zöliakie mit anderen Autoimmunerkrankungen, wie Diabetes mellitus Typ I oder Hashimoto-Thyreoiditis, und die daraus resultierende hohe Wahrscheinlichkeit, bei solchen Patienten eine Zöliakie zu diagnostizieren. Therapeutisch blicken wir gespannt auf die kommenden Jahre: Wird eine „Enzymtherapie“ kommen, durch die die immunologisch relevanten Gliadinsequenzen verdaut werden, noch bevor sie in der Dünndarmmukosa immunologisches Unheil anrichten? Werden wir genetisch Prädisponierte gegen Zöliakie impfen können? Bis dahin bleibt jedoch die glutenfreie Diät die einzige etablierte Therapieform der Zöliakie.

Vor diesen neuen Entwicklungen ist die aktuelle Ausgabe der Zeitschrift Der Gastroenterologe dem Schwerpunkt Zöliakie gewidmet und es wird dabei zusätzlich auf komplizierte Verläufe und das Thema der intestinalen Lymphome eingegangen:

Im 1. Artikel führen Schumann, Daum und Siegmund in den aktuellen Stand der Pathophysiologie der Zöliakie ein. Die Zöliakie als eine „ein- und ausschaltbare"Autoimmunerkrankung mit Gluten in der Funktion als Schalter zu begreifen, hat bereits viele Forschergruppen fasziniert und eine große Zahl an Erkenntnissen zur Folge, die nicht nur zum Verständnis der Erkrankung Zöliakie beigetragen hat, sondern uns auch das mukosale Immunsystem als Ganzes besser verstehen lässt. Klaus-Michael Keller beschreibt im folgenden Artikel die Zöliakie im Kindesalter. Gedeih- und Reifestörungen sind hier wichtige und für diese Altersgruppe spezifische Aspekte. Zudem wird hier spezieller Wert auf die Diagnostik beim Kind gelegt. Der Artikel von Felber und Stallmach geht explizit auf die eingangs ausgeführte Problematik ein: Was ist zu beachten, um auch die oligosymptomatischen Verläufe einer Zöliakie zu identifizieren? Ebenso findet sich hier eine differenzierte und leitlinienbasierte Diskussion zur sicheren und effizienten Diagnostik bei Verdacht auf Zöliakie. Bewusst sehr alltagsnah und daher im besonderen Sinne praxisrelevant ist der Artikel der Er- 
nährungswissenschaftlerin Nina Ludwig gehalten, der uns Medizinern - auch anhand von Beispielen - die Grundlagen der glutenfreien Diät näherbringen soll. Mag es doch für viele Patienten bei Erstdiagnose ein Schockerlebnis sein, in dessen Rahmen viele „diätetische Verbote“ ausgesprochen werden, ist es umso wichtiger, Alternativen aufzeigen zu können. Diese vermitteln dem Patienten gleich von Beginn an, dass er die Kostumstellung durchaus bewältigen kann. Dabei kann natürlich auch auf die sehr gute Prognose der Erkrankung bei strikter Einhaltung der glutenfreien Diät eingegangen werden.

Nur bei einem sehr kleinen Prozentanteil der Patienten entwickelt sich im weiteren Verlauf, gelegentlich auch primär, d. h. bei Erstdiagnosestellung, eine diätrefraktäre Zöliakie, die das Thema des nachfolgenden Artikels von Daum, Schumann und Siegmund ist. Hier ist es in erster Linie für den behandelnden Arzt wichtig, so wachsam zu sein, dass diese Diagnose möglichst zügig erkannt und dann in den entsprechenden Klinikzentren subkategorisiert und therapiert wird, da ein relevanter Anteil der Patienten an einem sehr schwer zu therapierenden TZell-Lymphom erkranken kann. Ein Thema, das sicherlich vor 10 Jahren noch nicht auf der Agenda eines solchen Hefts gestanden hätte, ist die Nicht-ZöliakieWeizensensitivität, in die Barmeyer und Ullrich einführen. Die letzten Jahre haben gezeigt, dass es offensichtlich ein großes Patientenkollektiv gibt, das z. T. zöliakieähnliche Symptome aufweist, bei dem zu einem beträchtlichen Teil jedoch ein extraintestinaler klinischer Befund mit Konzentrationsstörungen, Kopfschmerzen oder Hautveränderungen im Vordergrund steht, der auf eine glutenfreie Diät anspricht. Interessanterweise sind hier die zöliakiespezifischen Biomarker (Zöliakieserologie und Histologie der duodenalen Mukosa) unauffällig. Einziges Diagnostikum (und gleichzeitig einzige Therapieform) ist daher aktuell das Ansprechen auf eine glutenfreie Diät. Der letzte Artikel dieser Serie führt in die Welt der intestinalen Lymphome ein und vermag auf eine sehr gut strukturierte Art und Weise, sowohl die Lymphome zu kategorisieren als auch ihre Klinik und die zur Diagnosestellung notwendigen Untersuchungen aufzuzeigen. Abschließend erhält der Leser dieses Artikels einen Leitfaden zur Differenzialtherapie der Lymphome.

Wir glauben daher, Ihnen mit der aktuellen Ausgabe einen nahezu kompletten Überblick über ein sehr spannendes Feld unseres Fachs bieten zu können, und wollen uns an dieser Stelle für die vollkommen komplikationsfreie Mitarbeit der beteiligten Autoren zum einen und des redaktionellen Springer-Teams zum anderen an diesem Heft bedanken. Wir wünschen Ihnen viel Freude beim Lesen der Artikel und das Sammeln möglichst vieler neuer Erkenntnisse!

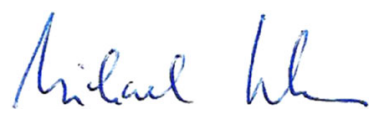

Dr. Michael Schumann

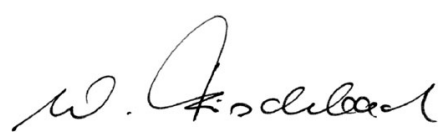

Prof. Dr. Wolfgang Fischbach

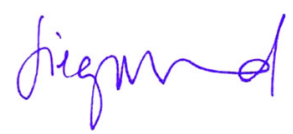

Prof. Dr. Britta Siegmund

\section{Korrespondenzadresse}

\section{Dr. M. Schumann}

Medizinische Klinik I m.S. Gastroenterologie, Infektiologie und Rheumatologie, Charité -

Universitätsmedizin Berlin, Campus Benjamin Franklin

Hindenburgdamm 30, 12200 Berlin,

Deutschland

michael.schumann@charite.de

\section{Prof. Dr. med. W. Fischbach}

Medizinische Klinik II, Klinikum AschaffenburgAlzenau

Am Hasenkopf, 63739 Aschaffenburg,

Deutschland

med2-aschaffenburg@t-online.de

\section{Prof. Dr. B. Siegmund}

Medizinische Klinik I m.S. Gastroenterologie, Infektiologie und Rheumatologie, Charité Universitätsmedizin Berlin, Campus Benjamin Franklin

Hindenburgdamm 30, 12200 Berlin,

Deutschland

britta.siegmund@charite.de

Interessenkonflikt. M. Schumann, W. Fischbach und $B$. Siegmund geben an, dass kein Interessenkonflikt besteht.

\section{Literatur}

1. Laass MW, Schmitz R, Uhlig HH, Zimmer KP, Thamm M, Koletzko S (2015) The prevalence of celiac disease in children and adolescents in Germany - results from the KiGGS study. Dtsch Arztebl Int 112:553-560

2. Rampertab SD etal (2006) Trends in the presentation of celiac disease. Am JMed 119(4):355.e9-e14

3. TheNPDGroup/Dieting Monitor, 52 weekdata, https://www.npd.com/wps/portal/npd/us/news/pressreleases/consumers-generally-view-gluten-freediet-as-healthy-for-everyone-reports-npd/ . Zugegriffen:28.5.2014 
Hier steht eine Anzeige.

刑 Springer 